\title{
Exploring the Path of Medical Services of Family Doctors for the Elderly from Perspective of "Combination of Medical and Health Care"
}

\author{
Zhang yumeng \\ Shaanxi Normal University \\ SNNU \\ Xi'an, China \\ 623580840@qq.com
}

\begin{abstract}
With the improvement of China's comprehensive national strength and the continuous development of medical technology, the average life expectancy of human beings has prolonged, and the problem of population aging has followed. Due to the large number of diseases and the chronic diseases of aging population, this feature poses a challenge to the medical health services for the elderly in China. This paper first analyzes the elderly medical service system in China, the family doctor service and China's limitations. It proposes to provide space for family doctors to participate in the medical services for the elderly in the context of "combination of medical care and healthcare", but finds that the service still has many shortcomings. Therefore, the exploration path of family doctors' integration into the elderly medical services is proposed from the four levels of policy, finance, technology and culture.
\end{abstract}

Keywords-Combination of medical and health care; Family doctor; The elderly; Medical service

\section{INTRODUCTION}

Since the 21 st century, with the continuous enhancement of China's comprehensive strength, the continuous development of medical technology, the extension of human life expectancy, China's aging problem has begun to become increasingly serious. According to experts' predictions, by 2025, China's elderly population will reach 300 million. In 2053, the elderly population will reach a peak of 487 million, accounting for $34.9 \%$ [1]. In China, there are problems such as large population base, uneven distribution, and uneven development among regions among the elderly. All of these pose great challenges to China's existing pension model. In addition, the elderly have a wide variety of diseases and are mainly chronic non-infectious diseases characterized by slow treatment, long cycle and high cost. As a result, the patient's psychological burden and family burden are heavy. Therefore, it is urgent for China to formulate a set of medical guidance policies combining old-age care and medical care.

In response to the above problems, in 2013, China first proposed the concept of combining medical care with old-age care in the "Several Opinions on Accelerating the Development of the Aged Care Service Industry". The purpose is to promote the integration of medical care and promote the cooperate of medical institutions and old-age care institutions. The combination of medical and health care first originated in Qingdao, and in 2002 began to explore and gradually formed the "family bed" system [2]. In order to meet the medical care needs of the elderly, the current health care system urgently needs a medical service role that can meet the daily medical needs of the elderly. The "family doctor" can provide timely and convenient medical services, which satisfies the elderly well. Therefore, family doctors should be promoted to integrate medical services with the elderly.

Based on the above background, this paper intends to explore the following issues, including: (1) the status quo of medical services for the elderly and family doctor services; (2) the space for family doctors to participate in the medical services for the elderly from the perspective of "Combination of medical and health care"; (3) The path of family doctors services for the elderly.

\section{PROGRESS OF MEDICAL SERVICES FOR THE ELDERLY IN CHINA}

As the age continues to increase, the physiological function of the elderly will continue to decline, so the elderly have different levels of potential demand for medical services. China's population aging has entered a period of rapid development. According to the data compiled by the China Business Research Institute published by the National Bureau of Statistics, as of the end of 2017, China's population over 65 years old reached 158.31 million, accounting for $17.3 \%$ of the country's total population [4] (Figure 1). Due to the rapid growth and large scale of the elderly population, the demand for medical and health service services will inevitably rise. However, the development of the elderly medical service industry in China is relatively slow, which makes the medical and nursing problems of the elderly become more and more prominent [5] 


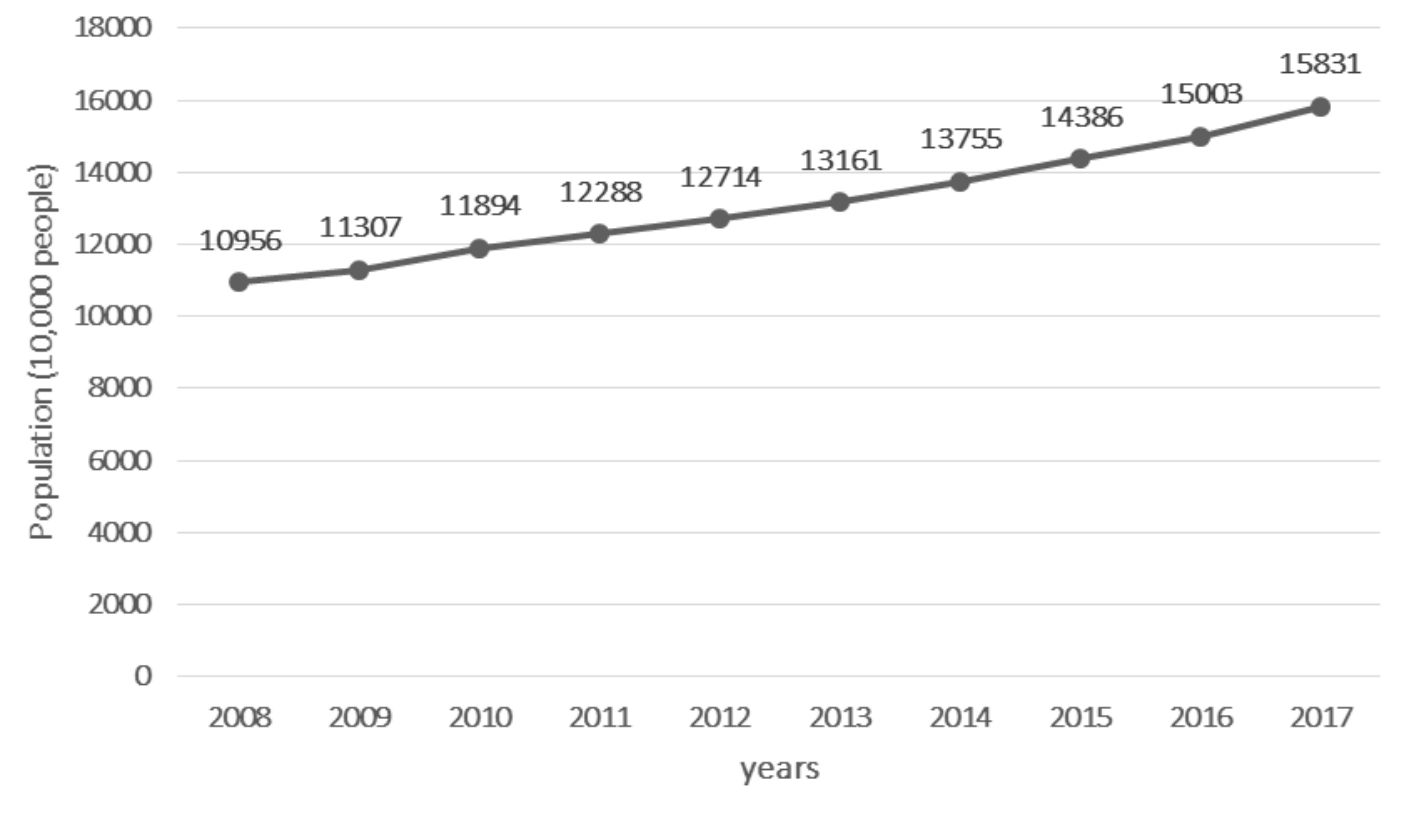

Fig. 1. Statistics on the number of elderly people aged 65 and over in China from 2008 to 2017

However, at present, in terms of expenses, the utilization and cost of medical services for the elderly are relatively high, and the medical expenses for the elderly are continuously increasing [6]. In terms of distance, community hospitals are far away from their places of residence due to inconvenient transportation. Older people need to spend a long time purchasing daily medicines or seeking medical treatment, while older people cannot afford to travel long distances [7-8]; In terms of service quality and human capital, the human resources structure of community health service organizations is unreasonable, the medical staff has a low level of business, and lacks professional health care knowledge. At the same time, the community provides a single pension service, mainly based on domestic services, and the elderly medical services are in short supply [9]. The public investment, construction, maintenance and supply of medical services for the elderly in rural areas are obviously insufficient. Medical facilities are not perfect, medical conditions are not good enough, medical services are not adequately supplied, and medical services are inconvenient [10].

Therefore, there is an urgent need for a role that can provide convenience and speed to meet the needs of the elderly for medical services. The "family doctor" is the best solution to meet this demand.

\section{FAMILY DOCTOR SERVICE SYSTEM AND CHINA'S LIMITATIONS}

The family doctor service is to manage the health of each family member through the community from the general practitioner to the family member. By signing the contract, the continuity, safety and effectiveness of the contracted family medical service is guaranteed. It is a comprehensive medical and health service mode and health management mode.
In order to solve the health service needs of the elderly in Japan, we have built a 24-hour home medical care system, providing on-site medical treatment activities and regularly patrolling, and can provide on-site services at any time [11]. The United States has established a comprehensive care service for the elderly, PACE, which provides services through the employment of long-term contracted staff to form a relatively stable working group. The staff will work with the contractor and their family members to develop a medical service plan [12]. Older people in Sweden can choose a doctor in the nearest hospital as a fixed family doctor. The general disease is treated by a family doctor in the hospital where the disease is located. If you see a specialist, it is recommended by a family doctor at the local hospital [13].

It can be seen from the above countries that "family doctors" is a method that is very conducive to solving the problems of health care services for the elderly in China. Therefore, China began to introduce the concept of general practitioners in the $1980 \mathrm{~s}$, and then gradually embarked on the road of exploring family doctors. Since the 1980s, some scholars have started research on family doctors. Some elderly people have obtained very convenient, timely and preferential medical services at family doctors, which has reduced the burden of diagnosis and treatment of large hospitals and reduced medical expenses. Family doctors, appointment rates, referral rates, and referral rates are slowly increasing; residents in the pilot areas have a certain understanding of family doctors, and more and more communities are conducting family doctor services [14].

However, China lacks policy support to guide the elderly to seek medical care, which restricts the development of family doctor services. At present, the policies for guiding medical treatment, such as grading diagnosis and treatment, two-way referral, etc., are all in the initial stage. The government has no policy to require the elderly in the community to seek medical 
treatment for the first time in community hospitals, the elderly are free to seek medical treatment and are only willing to choose to seek medical treatment from an expert they know or a well-known expert. This restricts the development of family doctors' signing model to a certain extent, and is also not conducive to the solution of medical problems for the elderly when they are living at home.

Therefore, China needs a policy that can combine the medical services for the elderly with family doctors. Through the family doctor service team, the elderly can be guided to seek medical treatment and the medical services for the elderly can be improved. Therefore, China has put forward the policy of "combination of medical and health care".

IV. THE SPACE FOR FAMILY DOCTORS TO PARTICIPATE IN THE HEALTH SERVICES FOR THE ELDERLY FROM PERSPECTIVE OF "COMBINATION OF MEDICAL AND HEALTH CARE"

The "combination of medical and health care" proposed by China refers to the combination of medical service resources and old-age resources to achieve optimal allocation of resources. General Secretary Xi Jinping pointed out: "We must build a pension service system based on home-based, community-based, institutional-replenishment, and medical care. Through the development model of medical and pension integration, medical rehabilitation and life care will be integrated. Modern medical service technology is combined with the old-age security model, and the medical service institutions and the aged care service institutions are functionally integrated to meet the health needs of the elderly and achieve healthy ageing."

Under the support of "combination of medical and health care", China has proposed to integrate medical care into the community and into the family. It mainly relies on the community health service system to promote family doctor services and provides on-site medical services for the elderly who sign up for the community. This policy provides a lot of room for family doctors to participate in the elderly medical services.

\section{A. The "combination of medical and health care" policy supports family doctors to participate in medical services for the elderly}

The purpose of "combination of medical and health care" is to integrate medical resources and provide a basic institutional basis for meeting the medical needs of the elderly. It proposes: "Promoting medical services to the community and even families, that is, promoting the development of medical institutions centered on community health services, and providing convenient medical care for the elderly in the community by signing family doctors for the elderly in the community."

\section{B. The "combination of medical and health care" policy provides human resources support for family doctors.}

Through the "combination of medical and health care" policy, more community health care workers can be involved in the family doctor's service team to provide more human support for the elderly medical services.

\section{The "combination of medical and health care" policy provides financial support for family doctors}

The human resources, financial resources and medical resources of the primary medical institutions are limited, but the community has a large amount of resources, but the two parties are their own masters, and they are not well integrated to achieve regional sharing of resources. Through the policy of "combination of medical and health care", it is possible to effectively integrate resources and provide a guarantee for family doctors to better participate in the medical services for the elderly.

With the support of all aspects of the policy of "combination of medical and health care", the community doctors-centered family doctor contracting system has been introduced. Older people can obtain more stable and convenient medical services through contracting family doctors, and the elderly can use them. The community's medical service resources make it easier for the elderly at home to see a doctor.

\section{THE PATH OF FAMILY DOCTORS PARTICIPATING IN MEDICAL SERVICES FOR THE ELDERLY FROM PERSPECTIVE OF "COMBINATION OF MEDICAL AND HEALTH CARE"}

In the context of "combination of medical and health care" policy, the family doctor-type medical service model provides a more stable, convenient and fast service for the elderly medical services, but because the service model still has many shortcomings. Therefore, it is necessary to improve from the aspects of policy, finance, technology and culture. So this article proposes the following solution path:

\section{A. Policy path}

The signing of family doctors by the elderly is the best "combination of medical and health care" model [15]. China should vigorously develop family doctor services and formulate family doctor development policies in line with geographical characteristics so that the regions across the country can develop in a balanced manner. Formulate policies to actively guide the masses to seek medical treatment reasonably. First, the first diagnosis should be conducted in a community hospital or through a family doctor to improve the graded diagnosis and treatment system in China.

\section{B. Financial path}

Improve the reimbursement mechanism of medical insurance, the project of family doctor services can be included in the scope of medical insurance reimbursement, the minimum fee for the proportion of medical expenses paid by the outpatient clinics of grassroots medical institutions, and the gap in the proportion of medical insurance reimbursement of medical institutions at different levels. Set up special funds to 
train general practitioners and compensate family doctors for their income.

\section{Technical path}

Expand the training channels for family doctors and enrich the family doctors. Improve the accessibility of family doctors, encourage general practitioners to work at the grassroots level, and increase the rate of family doctors in rural areas. Increase the introduction of general practitioners at the grassroots level, encourage grassroots medical institutions to recruit general practitioners, and improve the number and overall quality of general practitioners through on-the-job training and rehiring of retired doctors.

\section{Cultural path}

Do a good job in family doctors to raise awareness. Unsigned people can actively learn about the latest policies of family doctors, change their medical thinking, and obey the grading diagnosis and treatment policy. The contracted people can actively promote the family doctor service and improve the utilization rate of family doctor services, so that the family doctor service can benefit more people.

\section{CONCLUSION}

In short, with the aging of China's population, the "combination of medical and health care" model of old-age care is a model that the elderly need urgently and the government needs to implement. In different forms of "combination of medical and health care", the provision of community primary medical services through the signing of family doctors should be the focus of the task of "combination of medical and health care". Family doctors have a wide coverage, which helps the elderly to care for the elderly and reduce the psychological and living burden of the elderly. This form of "combination of medical and health care" is most in line with the needs of the elderly, so family doctors should be vigorously developed, and the form of contracted family doctors is also the best form of "combination of medical and health care".

\section{REFERENCES}

[1] "China's "Thirteenth Five-Year" period will focus on complementing the short-board development of rural ageing," Xinhuanet:http://www.xinhuanet.com/politics/201603/29/c_1118480164 .htm, March 29, 2016.(in Chinese)

[2] Zhu Fengmei, Miao Ziqiang. The Connotation, Current Situation and Dilemma of "Combination of Medicine and Health" under the Background of Aging[J]. China Health Economics, 2018, 03: 11-5.(in Chinese)

[3] "National Health Planning Commission: "There are four modes of medical integration", Health World: http://www.cnhealthcare.com/article/2015 0512/content-473761.html, May 12, 2015. (in Chinese)

[4] National Bureau of Statistics of the People's Republic of China. Statistical Communiqué of the 2017 National Economic and Social Development of the People's Republic of China [EB /OL].( 2018 - 02 28) [2018 - 04- 18]. ]http: / /www. stats.gov .cn /tjsj /zxfb /201802 /t20180228_1585631. html. (in Chinese)

[5] Xing Mei. Research on social pension service in China at the present stage [D]; Jilin University, 2014. (in Chinese)

[6] Li Fen, Ding Lingling, Wang Linan, et al. Analysis of the needs, utilization and cost characteristics of medical services for the elderly in Shanghai [J]. Health Economics Research, 2018, 06: 26-9. (in Chinese)

[7] Li Min, Jin Kaiwei, Liu Huafu. Daily Medical Treatment and Satisfaction of Rural Elderly in Mountainous Areas [J]. Chinese Journal of Gerontology, 2018, 04: 954-5. (in Chinese)

[8] Li Wei. Community Health Service Center of "Over-Huaiwei" — A Survey of Rural Medical and Health Service Models in Beijing [J]. Capital Medicine, 2009, 21: 18-20. (in Chinese)

[9] Lu Zequn, Zuo Linkai, Yuan Wei, et al. Investigation on the status quo and needs of community aged care services in Wuhan [J]. Journal of Community Medicine, 2017, 15: 83-4. (in Chinese)

[10] Yang Wenming, Qi Haimei, Qiu Yien, et al. Vigorously improving rural medical services for the elderly [J]. Social Governance, 2017, 05: 33-6. (in Chinese)

[11] Tian Xianglan. Reform of medical care supply system and integration of medical care in Japan [J]. Journal of Japanese Studies, 2017, 04: 61-8. (in Chinese)

[12] Yang Xiaojuan, Ding Hansheng, Du Lixia. Comprehensive Care Service Model for the Elderly in the United States and Its Enlightenment [J]. China Health Resources, 2016, 04: 354-7. (in Chinese)

[13] Wang Lujiao, Zhao Liang. The current situation of social medical care for the elderly in Sweden and its reference [J]. Foreign Medical Sciences (Medical Geography), 2017, 03: 218-21. (in Chinese)

[14] Dong Yuming, Du Xueping, Dong Jianqin. Feasibility Survey of Family Doctors' First Diagnosis System in Beijing Yuetan Community[J]. Chinese Journal of General Practice, 2009, 09: 763-5. (in Chinese) 